Epilepsy due to Sturge-Weber is presented. The patient presented to the ED with acute confusion, eye deviation and right hemiparesis similar to two previous admissions. 1tm-eeg showed diffuse slowing. DWI obtained within 72-hr showed no ischemic changes, analysis of the EEG nfraslow (ISA) activity was undertaken using LFF 0.01 AND HFF of $0.1 \mathrm{~Hz}$ respectively. Results: Continuous subclinical unilateral rhythmic ictal ISA was identified. This was only evident in the left hemisphere which correlated with the structural changes due to SWS. A trial of continuous IV midazolam resulted in immediate resolution of the contralateral hemiparesis and encephalopathy. Conclusion: Continuous prolonged rhythmic ictal Infraslow activity (ISA) can cause super-refractory subclinical focal status epilepticus. This has not previously reported, and we propose this should be called Infraslow status epilepticus (ISSE). ISA analysis should be performed in all patients with unexplained subclinical status epilepticus.

\section{Neurology (General Neurology)}

\section{P.024}

Cauda equina syndrome secondary to leptomeningeal carcinomatosis of gastroesophageal junction cancer

\section{A Alkhotani (Makkah)*N Alrishi (Makkah) M Alhalabi (Makkah)}

doi: $10.1017 / \operatorname{cjn} .2015 .135$

Background: Leptomeningeal Carcinomatosis (LMC) is defined as a diffuse or multifocal malignant infiltration of the Pia matter and arachnoid membrane. It is clinically diagnosed in $5-10 \%$ of all cancer patients. The most commonly reported cancers associated with LMC are breast, lung and hematological malignancies. Patients with LMC commonly present with multifocal neurological symptoms. Symptoms are related to increase intracranial pressure, hemispheric dysfunction, cranial neuropathies and spinal roots dysfunction. We report a case of LMC secondary to Gastroesophageal junction cancer present initially with Cauda equine syndrome. Methods: A 51 year old male patient with adenocarcinoma of Gastroesophageal junction who underwent surgical resection, chemotherapy and radiation therapy. Nine month after diagnosis he presented with left leg pain, mild weakness and saddle area numbness. Initial radiological examination were unremarkable. Subsequently he had worsening of his leg weakness, fecal incontinence and urine retention. Two days later he developed rapidly progressive cranial neuropathies including facial diplegia, sensorineural hearing loss, dysarthria and dysphagia. Results: MRI with and without contrast showed diffuse enhancement of leptomeninges surrounding the brain, spinal cord and Cauda equine extending to the nerve roots. Cerebrospinal fluid cytology was positive for malignant cells. Conclusion: In cancer patient with Cauda equina syndrome and absence of structural lesion on imaging, LMC should be considered.

\section{P.025}

Intravascular large B-Cell lymphoma (IVLBCL) presenting as lumbosacral polyradiculopathy

\section{SR Peters (Montreal)* Z Dastani (Montreal) CH Chalk (Montreal)}

doi: 10.1017/cjn.2015.136

Background: IVLBCL is potentially treatable but difficult to diagnose. Methods: A case of progressive leg weakness and sphincter dysfunction diagnosed only at autopsy. Results: A 72 year old female presented with three weeks of increasing leg weakness and three days of urine and stool incontinence. Electrophysiological testing showed reduced tibial and peroneal CMAPs, preserved sural SNAPs, and bilateral gastrocnemius fibrillations, consistent with a pre-ganglionic lesion. Lumbosacral MRI with gadolinium showed no abnormalities. CSF was examined three times (protein 1.18-1.25, glucose 2.6-3.1, normal cell count and cytology). Whole-body FDG PET scanning showed hypermetabolic foci at the tongue base and in the mediastinum, but biopsy of both revealed no abnormality. Leg weakness progressed over three months and spread to the arms despite a course of IVIg. Four months later she died of cardiorespiratory arrest. Autopsy revealed the presence of large atypical B-cells within the lumen of small and medium sized vessels in numerous organs. There was evidence of anterior spinal artery obstruction with lymphocytes and anterior horn infarction in the lumbar cord. Conclusions: Although the literature reports that IVLBCL responds well to chemotherapy, this patient illustrates the difficulty of ante-mortem diagnosis. Nerve root biopsy may be warranted in such patients.

\section{P.026}

A prospective 1-year study of postural tachycardia and the relationship to non-postural versus orthostatic symptoms

J Baker (London)* IS Palamarchuk (London) K Kimpinski (London) doi: 10.1017/cjn.2015.137

Background: Postural tachycardia syndrome (POTS) is defined as heart rate (HR) increments $\geq 30 \mathrm{bpm}$ on head-up tilt (HUT) associate with orthostatic symptoms (lightheadedness, dizziness, palpitations, etc.). A large proportion of asymptomatic young adults naturally express excessive HR increments on HUT, which has raised questions regarding whether revision to the diagnostic criteria for POTS in younger patient populations is needed. In addition, poor adaptation to environmental stressors may contribute to the disability experienced by POTS patients. Objectives: 1) determine whether asymptomatic patients were predisposed to developing constitutional symptoms that could result in the full syndrome of POTS, and 2) determine whether these symptoms correlated to postural HR or orthostatic symptoms. Methods: HR response to HUT and orthostatic and constitutional symptoms in 30 asymptomatic POTS patients were evaluated after 1 year. Results: HR increment at follow-up demonstrated no correlation with general fatigue $(\mathrm{r}=0.006)$, body vigilance $(r=0.195)$, physical $(r=-0.087)$ and mental $(r=-0.137)$ health, or orthostatic scores $(r=0.04)$. Orthostatic scores significantly correlated with general fatigue $(r=0.374)$ and body vigilance $(r=0.392)$ $(\mathrm{p}<0.05)$. Conclusion: These data support that the majority of young individuals express benign orthostatic tachycardia and further argue for re-evaluation of the HR criteria for diagnosing POTS in younger populations. 